\title{
Attenuation of PERK enhances glucose- stimulated insulin secretion in islets
}

\author{
Min Joo Kim, Se Hee Min', Seon Young Shin², Mi Na Kim², Hakmo Lee², Jin Young Jang³, Sun-Whe Kim², \\ Kyong Soo Park ${ }^{1,2}$ and Hye Seung Jung ${ }^{1,2}$
}

'Department of Internal Medicine, Seoul National University College of Medicine, Seoul, Republic of Korea IInnovative Research Institute for Cell Therapy, Seoul, Republic of Korea

3Department of Surgery, Seoul National University College of Medicine, Seoul, Republic of Korea

Correspondence should be addressed to H S Jung: junghs@snu.ac.kr

\section{Abstract}

PERK is a pancreatic endoplasmic reticulum (ER) kinase. Its complete deletion in pancreatic $\beta$ cells induces insulin deficiency; however, the effects of partial Perk suppression are unclear. We investigated the effect of partial PERK suppression using the specific PERK inhibitors GSK2606414 and GSK2656157. Low-dose GSK2606414 treatment for $24 \mathrm{~h}$ enhanced glucose-stimulated insulin secretion (GSIS), islet insulin content and calcium transit in mouse (at $40 \mathrm{nM}$ ) and human (at 50-100 nM) pancreatic islets. GSK2606414 also induced the expression of the ER chaperone BiP and the

\section{Key Words}

- endoplasmic reticulum

- BiP

- calcium

- insulin

- pancreatic islets

- PERK kinase release of calcium from the ER. When Bip expression was inhibited using a Bip siRNA, the GSK2606414-induced augmentation of the ER calcium level, islet insulin contents, glucose-stimulated cytosolic calcium transit and GSIS were abrogated. In both wild-type and insulin-deficient Atg7-knockout mice, 8 weeks of GSK2656157 treatment enhanced GSIS and improved hyperglycemia without affecting body weight. In conclusion, partial PERK inhibition induced BiP expression in islets, increased glucose-stimulated calcium transit and islet insulin contents and enhanced GSIS, suggesting that low-dose PERK inhibitors could potentially be used to treat insulin deficiency.

Journal of Endocrinology (2018) 236, 125-136

\section{Introduction}

Eukaryotic translation initiation factor 2-alpha kinase 3 (also known as pancreatic endoplasmic reticulum kinase, PERK) is a transmembrane protein located in the endoplasmic reticulum (ER). PERK senses ER stress from its ER luminal domain, changes its oligomerization state and transduces the stress signal into the cytoplasm via autophosphorylation of its cytosolic kinase domains, which results in the subsequent phosphorylation of eukaryotic translation initiation factor 2A (EIF2A). ER stress is caused by the accumulation of unfolded proteins in the ER, which triggers the so-called unfolded protein response (UPR), consisting of the attenuation of translation, the transcription of ER chaperones and the upregulation of ER-associated degradation (ERAD). The UPR helps to maintain cellular homeostasis, but too much UPR can be harmful to cells (Kaufman 2002).

Pancreatic $\beta$ cells are considered to be under high ER stress, because a large amount of insulin is synthesized and processed in the ER. Therefore, a failure to regulate ER stress or the UPR can induce $\beta$ cell dysfunction and death (Back \& Kaufman 2012). PERK function has been reported to have important implications in $\beta$ cells. In humans, several loss-of-function mutations of PERK have been shown to induce severe insulin deficiency 
and neonatal diabetes mellitus (DM), known as WolcottRallison syndrome (Delepine et al. 2000). Perk-knockout $\left(\right.$ Perk $\left.^{-1-}\right)$ mice have severe defects in fetal/neonatal $\beta$ cell proliferation and differentiation along with impaired insulin trafficking and cell survival (Harding et al. 2001, Zhang et al. 2002, 2006, Gao et al. 2012).

Perk heterozygous $\left(\right.$ Perk $\left.^{+-}\right)$mice have demonstrated different phenotypes. Initially, they were reported to be slightly glucose intolerant (Harding et al. 2001); however, Wang and coworkers found that Perk ${ }^{+-}$mice exhibited enhanced insulin secretion during neonatal and juvenile development, resulting in a transient reduction in blood glucose levels (Wang et al. 2014). In addition, a partial reduction of the Perk gene dosage in Akita mutant diabetic mice increased pancreatic insulin contents and improved blood glucose levels, while an increase of the Perk gene dosage had the opposite effects (Gupta et al. 2010). The authors concluded that the suppression of Perk downregulated the activated ERAD in the Akita mice, resulting in less degradation of insulin.

The previous results suggest that the effects of Perk suppression on $\beta$ cells could vary greatly according to the degree of Perk suppression and the cellular environment. Therefore, we investigated the effects of different degree of PERK inhibition on $\beta$ cell function using specific PERK inhibitors (PERKi) in vitro and in vivo.

\section{Materials and methods}

\section{PERK inhibitors}

For the in vitro experiments, we treated isolated islets for $24 \mathrm{~h}$ with the PERK inhibitor GSK2606414 (Selleck, Houston, TX, USA) dissolved in DMSO (Sigma-Aldrich) (Axten et al. 2012).

For the in vivo mouse experiments, we dissolved GSK2656157 (Selleck), a PERK inhibitor that has been optimized for a preclinical setting (Axten et al. 2013), in $0.5 \%$ hydroxypropyl methyl cellulose and $0.1 \%$ Tween-80 in water $(\mathrm{pH}$ 6.75). Atkins and coworkers previously investigated the pharmacokinetics and pharmacodynamics of GSK2656157 and used dosages as high as $150 \mathrm{mg} / \mathrm{kg} /$ day in mice to completely inhibit PERK kinase activity (Atkins et al. 2013). From that report, we calculated the dose of GSK2656157 required to blood levels below $120 \mathrm{nM}$. Based on those calculations, we tested a dosage of 3-8 mg/kg GSK2656157 twice per day in preliminary experiments (Supplementary Fig. 1, see section on supplementary data given at the end of this article). We observed a trend of dose-dependent glucoselowering effect until a dosage of $5 \mathrm{mg} / \mathrm{kg}$ twice per day in a mouse model of insulin deficiency, so we selected that dosage as the most optimal one for the study.

\section{Animal studies}

We used C57Bl/6J mice (Biomedical Center for Animal Resource Development of Seoul National University, Seoul, Korea) and $A \operatorname{tg} 7 \mathrm{f} / \mathrm{f}: R^{2} p-\mathrm{Cre}^{+}$(Atg $7 \Delta \beta$ cell)-mutant mice with the $\mathrm{C} 57 \mathrm{Bl} / 6 \mathrm{~J}$ background in our experiments. We generated the $\operatorname{Atg} 7 \Delta \beta$ cell mice as previously described (Jung et al. 2008).

We randomized male mice at 10-12 weeks of age into vehicle and PERKi groups. The mice in the PERKi group $(n=10-12)$ received $5 \mathrm{mg} / \mathrm{kg}$ GSK2656157 twice per day by oral gavage for 8 weeks, and the mice in the vehicle group $(n=10-12)$ received equal volume of vehicle $(0.5 \%$ hydroxypropyl methyl cellulose and $0.1 \%$ Tween-80 in water). We measured the fed blood glucose levels and body weights of the mice every 2 weeks. After the 8 -week treatment, we fasted the mice overnight and performed a glucose tolerance test (GTT) by intraperitoneal injection of $1 \mathrm{~g} / \mathrm{kg}$ glucose followed by serial measurements of blood glucose. We obtained serum before and $30 \mathrm{~min}$ after the glucose loading to determine the serum insulin levels. On the next day, we fasted the mice for $6 \mathrm{~h}$ and then performed an insulin tolerance test (ITT) by intraperitoneal injection of $0.5 \mathrm{unit} / \mathrm{kg}$ insulin followed by serial measurements of blood glucose. We measured the blood glucose levels using a One-Touch Ultra glucometer (Johnson \& Johnson) and the serum insulin using an ELISA kit (ALPCO, Salem, MA, USA). All animal experiments were conducted in accordance with the Institutional Animal Care and Use Committee of Seoul National University Hospital (SNU-150327-3-2).

\section{Mouse islets}

We used the intra-ductal collagenase injection technique to isolate pancreatic islets from $\mathrm{C} 57 \mathrm{Bl} / 6 \mathrm{~J}$ mice around 18-25 weeks of age after an overnight fast (Jung et al. 2008). We cultured the isolated islet in RPMI medium (Welgene, Gyeongsan-si, Korea). For the siRNA experiments, we dissociated the mouse islets into single cells using trypsin (Thermo Fisher Scientific) and transfected cells with 120 nM Bip siRNA (siBip; Invitrogen) or negative control siRNA (siNS; Bioneer, Daejeon, Korea) using Lipofectamine RNAiMAX (Thermo Fisher Scientific) according to the manufacturer's instructions. 


\section{Human islets}

We obtained human pancreatic tissues from 14 individuals (three with DM and 11 without DM) who underwent elective pancreatic surgery. The study was approved by the Institutional Review Board of Seoul National University Hospital (IRB No. 0901-010-267). All the study participants provided written informed consent. The mean age of the participants was $60 \pm 4$ years. Half of the participants were male, and the mean BMI was $22.1 \pm 0.6 \mathrm{~kg} / \mathrm{m}^{2}$. The mean $\mathrm{HbA}_{1 \mathrm{C}}$ of the three participants with DM was $6.6 \pm 0.2 \%$.

We isolated islets from $0.41 \pm 0.05 \mathrm{~g}$ of pancreatic tissue from each participant that appeared normal upon gross inspection. We digested the pancreatic tissues with collagenase (SERVA Electrophoresis GmbH, Heidelberg, Germany) and separated the islets using Ficoll gradients centrifugation. After washing the separated islets, we hand-picked individual islets, counted them and incubated them in RPMI medium with 10\% FBS.

\section{Western blot}

We extracted total protein from homogenized islets or dispersed islet cells using RIPA buffer (BRI-9010, T\&I, Korea) mixed with a protease inhibitor cocktail and phenylmethylsulfonyl fluoride (PMSF). We boiled the protein samples in SDS sample buffer, separated them by SDS-polyacrylamide gel electrophoresis, and then transferred them to nitrocellulose membranes for immunoblotting. We detected the blots using the ECL Western Blotting Substrate (Thermo, \#NCI4080KR). We used the following primary antibodies: anti-p-PERK (Cell Signaling \#3179, 1:1000), anti-PERK (Cell Signaling \#3192, 1:1000), anti-p-EIF2A (Cell Signaling \#9721, 1:1000), anti-EIF2A (Cell Signaling \#9722, 1:1000), anti-BiP (Cell Signaling \#3177, 1:1000) and anti-ACTIN (Sigma-Aldrich A5441, 1:5000).

\section{Glucose-stimulated insulin secretion (GSIS) and islet insulin contents}

To measure GSIS, we fasted isolated islets or dispersed islet cells for $20 \mathrm{~min}$ in KRB HEPES (KRBH) buffer containing $0.2 \%$ BSA and low glucose $(2.8 \mathrm{mM}$ for mouse islets; $1.67 \mathrm{mM}$ for human islets) at $37^{\circ} \mathrm{C}$. We then replaced the media with fresh low glucose $\mathrm{KRBH}$, incubated the cultures for $40 \mathrm{~min}$, collected the supernatants and replaced the media with high glucose $(17.5 \mathrm{mM}) \mathrm{KRBH}$. After $40 \mathrm{~min}$ of further incubation, we collected the supernatants and assayed the insulin using an ELISA kit (ALPCO).
To adjust secreted insulin levels with the intraislet insulin contents, we collected the cells after GSIS, dissolved them in $0.2 \mathrm{M} \mathrm{HCl}$ in $95 \%$ ethanol and then sonicated them. We then neutralized the islet lysates with $0.2 \mathrm{M} \mathrm{NaOH}$, collected the supernatants and assayed the insulin using an ELISA kit (ALPCO). Secreted insulin amount (ng/10 islets/40 min) was divided by intra-islet insulin amount (ng/10 islets), and it was presented as \% change of control.

\section{Glucose-stimulated cytosolic calcium}

We incubated islet cells in low glucose KRBH containing Fluo-4 NW dye mix (Molecular Probes, Invitrogen) for $40 \mathrm{~min}$. We then traced the cellular calcium before and after stimulation with high glucose $\mathrm{KRBH}$ through a channel using the Infinite F200 PRO system (TECAN, Grödig, Austria), by measuring the fluorescence excitation and emission at $494 \mathrm{~nm}$ and $516 \mathrm{~nm}$, respectively. To assess the calcium transit from intracellular stores, we performed the same procedures using media from which the calcium has been removed and to which $0.1 \mathrm{mM}$ EGTA had been added.

\section{Estimation of ER calcium}

To measure the release of calcium from ER in response to carbachol treatment, we exposed islet cells to $200 \mu \mathrm{M}$ diazoxide and $20 \mathrm{mM}$ glucose for $40 \mathrm{~min}$ and then transferred the cells to Fluo-4 NW dye (Molecular Probes) mixed in KRBH buffer without calcium. We measured fluorescence excitation using the Infinite F200 PRO (TECAN) before and after stimulation with $250 \mu \mathrm{M}$ carbachol (Tocris Bioscience, Bristol, UK) (Wang et al. 2013).

To measure calcium leakage from the ER to the cytosol, we exposed islet cells to Fluo-4 NW in $10 \mathrm{mM}$ HEPES-KOH buffer containing $140 \mathrm{mM} \mathrm{NaCl}, 5 \mathrm{mM} \mathrm{KCl}$, $1 \mathrm{mM} \mathrm{MgCl}_{2}, 0.5 \mathrm{mM}$ EGTA and $10 \mathrm{mM}$ glucose (pH 7.35). We measured the fluorescence excitation before and after adding $1 \mu \mathrm{M}$ thapsigargin, which inhibits ER calcium refilling (Schorr et al. 2015).

\section{Reverse transcription polymerase chain reaction (RT-PCR)}

We extracted total RNA from islet cells using TRIzol (Invitrogen) and a total RNA extraction kit (Intronbio, Seongnam, Korea). We then performed reverse transcription using M-MLV Reverse Transcriptase 
Table 1 Primer sequences for RT-PCR.

\begin{tabular}{l}
\hline \\
\hline Atf4 \\
Bip \\
Chop \\
Edem \\
Erdj4 \\
Ero1b \\
Gapdh \\
Grp94 \\
Hrd1 \\
Spliced Xbp-1 \\
18s
\end{tabular}

Forward
CCT GAA CAG CGA AGT GTT GG
ACT TGG GGA CCA CCT ATT CCT
CCA CCA CAC CTG AAA GCA GAA
GCA ATG AAG GAG AAG GAG ACC C
GAA TTA ATC CTG GCC TCC AA
GGG CCA AGT CAT TAA AGG AA
AGT GGA GAT TGT TGC CAT CAA
AAT AGA AAG AAT GCT TCG CC
TGG CTT TGA GTA CGC CAT TCT
AGGAAACTGAAAAACAGAGTAGCAGC
AGTCCC TGC CCT TGT ACA CA
AGTCCC TGC CCT TGT ACA CA
Reverse
TGG AGA ACC CAT GAG GTT TCA A
ATC GCC AAT CAG ACG CTC C
TGA GAG CCA GAC GTG TTC GT
TAG AAG GCG TGT AGG CAG ATG G
CAG GGT GGT ACT TCA TGG CT
TTT ATC GCA CCC AAC ACA GT
TGG ATG CAG GGA TGA TGT TCT
TCT TCA GGC TCT TCT TCT GG
CCA CGG AGT GCA GCA CAT AC
TCC TTC TGG GTA GAC CTC TGG
GAT CCG AGG GCC TCA CTA AAC

(Promega). For quantitative PCR, we used Maxime PCR PreMix (i-StarTaq) (Intronbio) and the LightCycler 96 Real-Time PCR System (Roche). The primer sequences are presented in Table 1. We normalized the data with the mRNA of Gapdh and 18s.

\section{Statistics}

We expressed all values as the mean \pm S.E.M. We analyzed the glucose stimulation results, blood glucose and body weights by 2-way repeated-measures ANOVA. We analyzed the results of the siRNA experiments by 2-way ANOVA. We analyzed the results of the other experiments by Student's $t$-test or 1-way ANOVA according to the numbers of compared groups. In the case of samples with large variation, we used the paired $t$-test. For post hoc analyses, we used Bonferroni posttests. We considered $P$ values less than 0.05 to be statistically significant.

\section{Results}

\section{Partial reduction of PERK phosphorylation by low-dose GSK2606414-enhanced GSIS and islet insulin contents}

GSK2606414 inhibits PERK kinase activity by binding to the ATP-binding site in the cytoplasmic domain of PERK (Axten et al. 2012). A previous study showed that 1000 nM GSK2606414 completely inhibited PERK activity and acutely abrogated GSIS and calcium dynamics within 20 min (Wang et al. 2013). To achieve various degrees of chronic PERK inhibition, we treated mouse islets for $24 \mathrm{~h}$ with GSK2606414 concentrations ranging from $20 \mathrm{nM}$ to $1000 \mathrm{nM}$. GSIS was significantly increased at $40 \mathrm{nM}$ and tended to decrease at higher concentrations, even becoming suppressed at concentrations above $250 \mathrm{nM}$ (Fig. 1A, left panel).
In human islets, 24 -h treatment with $50 \mathrm{nM}$ or $100 \mathrm{nM}$ GSK2606414 enhanced GSIS whereas that with $150 \mathrm{nM}$ GSK2606414 did not (Fig. 1A, right panel). GSK2606414 also enhanced basal insulin secretion in human islets in the fasting state. GSK2606414 decreased the phosphorylated PERK on western blotting in a dosedependent manner and caused the downregulation of EIF2A phosphorylation (Fig. 1B). The treatment with 40nM GSK2606414 significantly increased the islet insulin contents (Fig. 1C). When the GSIS was adjusted with the islet insulin contents, the 40nM GSK2606414 treatment did not change the insulin levels during glucose stimulation (Fig. 1D, left panel), although it significantly increased the ratio of the high glucose-insulin level to the low glucose-insulin level (Fig. 1D, right panel). Those findings suggest that low-dose GSK2606414 increases not only the insulin contents of islets but also the insulin exocytosis in response to glucose stimulation, both of which could enhance GSIS.

\section{Low-dose GSK2606414 increased glucose- stimulated cytosolic calcium transit}

Cytosolic calcium is a major inducer of insulin exocytosis. We found that 40nM GSK2606414 enhanced glucosestimulated calcium transit in mouse and human islets (Fig. 1E).

\section{Low-dose GSK2606414 induced BiP expression}

Unlike PERK phosphorylation, EIF2A phosphorylation was not significantly reduced by $40 \mathrm{nM}$ GSK2606414 (Fig. 1B). Likewise, the $40 \mathrm{nM}$ GSK2606414 treatment did not significantly change the expression of Atf4, the downstream effector of EIF2A (Fig. 2A). We examined other UPR genes at the mRNA level and found that they were not significantly affected by low-dose GSK2606414; 

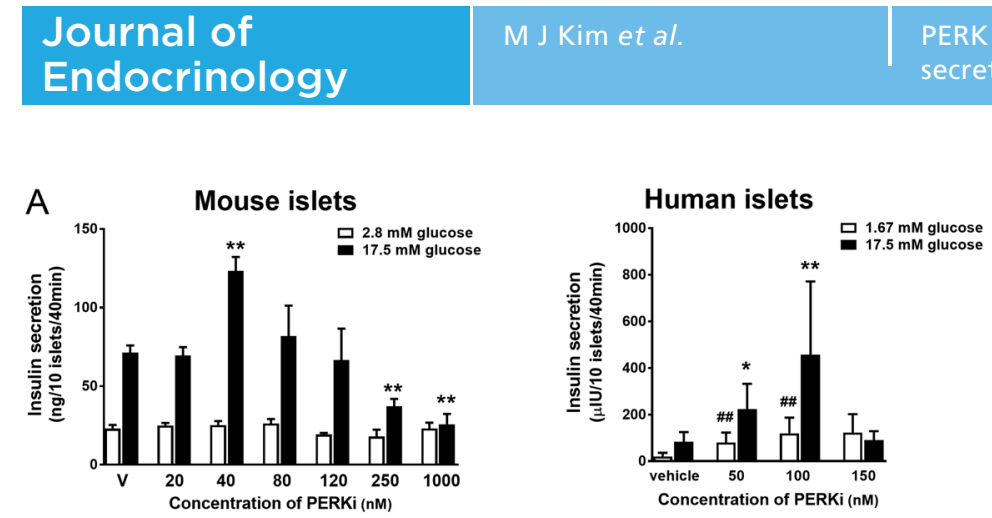
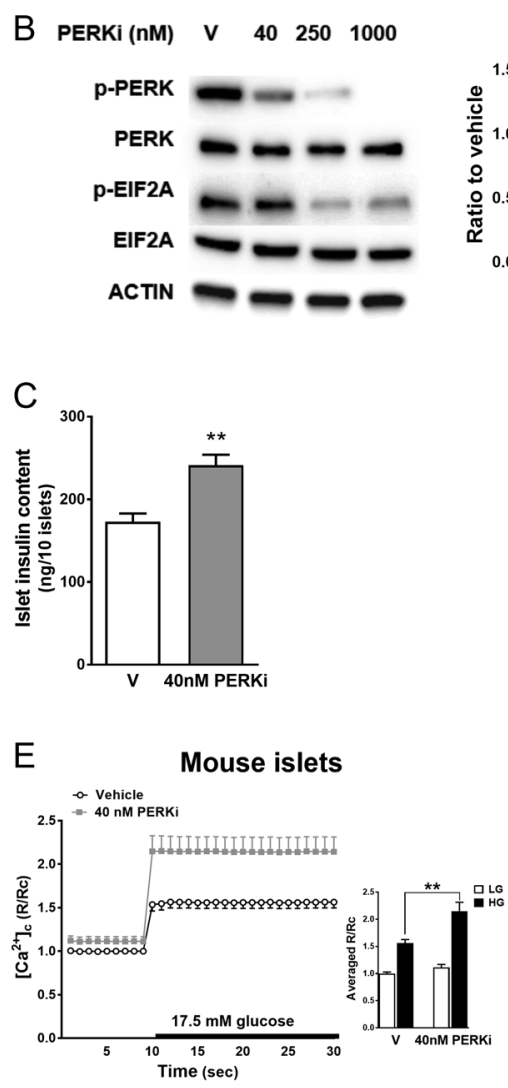
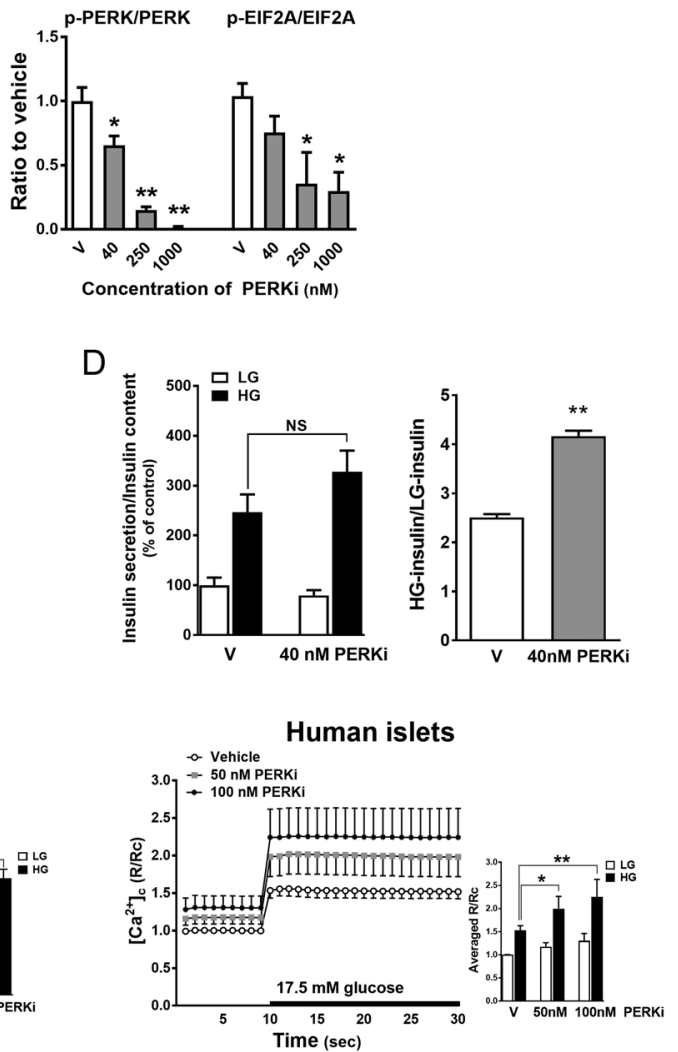

\section{Figure 1}

Partial reduction of PERK phosphorylation by low-dose GSK2606414 augmented GSIS, islet insulin contents and cytosolic calcium transit. GSK2606414, a specific synthetic PERK inhibitor, was treated for $24 \mathrm{~h}$ with isolated islets. (A) GSIS from mouse islets $(n=3-6)$ and human islets $(n=3-6)$ at various concentrations of GSK2606414. Logarithmic transformation was done before statistical analysis for the human islets. (B) A representative picture of western blotting for PERK and EIF2A using mouse islets according to the concentrations of GSK2606414, and the quantitative analyses $(n=3)$. (C) Islet insulin contents by $40 \mathrm{nM}$ of GSK2606414 ( $n=6)$. (D) GSIS adjusted by islet insulin contents (left panel), and the ratio of HG insulin to LG insulin (right panel, $n=6)$. (E) Glucose-stimulated calcium transit. Cytosolic calcium concentrations ( $R$ ) were expressed as relative ratios to the control before stimulation (Rc) $(n=11)$. For statistical analyses, the $R / R c$ measures were averaged as in the inset. Glucose-stimulation experiments were analyzed by 2-way repeated-measures ANOVA and Bonferroni posttests. For the others, Student's $t$-test and 1-way ANOVA with Bonferroni posttests were used. HG, high glucose; LG, low glucose; NS, no significant difference; PERKi, PERK inhibitor; $V$, vehicle. ${ }^{*} P<0.05 ;{ }^{*} P<0.01$ vs vehicles. however, Bip expression was significantly increased by $50 \%$ (Fig. $2 \mathrm{~A}$ ), and the BiP protein levels were also increased in mouse and human islets (Fig. 2B).

\section{Low-dose GSK2606414 induced glucose-stimulated \\ calcium release from intracellular calcium stores, along with increased ER calcium}

The ER chaperone BiP not only facilitates protein processing in the ER (Dudek et al. 2009) but also has a direct role in the control of the efflux of calcium ions from the ER (Dudek et al. 2015). We observed that $40 \mathrm{nM}$ GSK2606414 significantly increased glucose-stimulated calcium transit (Fig. 1E). Extracellular calcium is a major source of cytosolic calcium transit during GSIS, but intracellular calcium stores, including those in the ER, are also used (Henquin 2000, Straub \& Sharp 2002). We tested whether intracellular calcium stores contributed to the GSK2606414-induced effects on cytosolic calcium levels, by depleting the extracellular calcium in the media and prohibiting calcium influx using calcium-chelating EGTA. We found that even under those conditions, 40nM GSK2606414 significantly increased glucosestimulated calcium transit (Fig. 2C). Next, we stimulated hyperpolarized cells with carbachol in calcium-free media to assess ER calcium release through inositol trisphosphate receptor (IP3R) activation (Wang et al. 2013). GSK2606414 at $40 \mathrm{nM}$ increased the area under the curve (AUC) above each baseline level of cytosolic calcium (Fig. 2D), suggesting that the ER may function as an intracellular calcium stores during GSK2606414enhanced GSIS. 


\begin{tabular}{l|l|l|c|}
$\begin{array}{l}\text { Journal of } \\
\text { Endocrinology }\end{array}$ & $\begin{array}{l}\text { PERK inhibition increases insulin } \\
\text { secretion }\end{array}$ & $\mathbf{2 3 6 : 3}$ \\
\hline
\end{tabular}

A

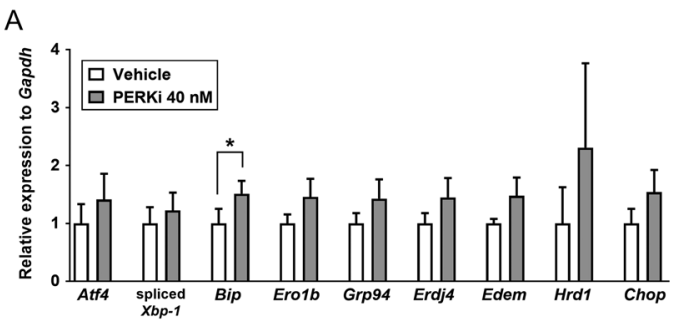

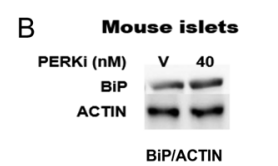
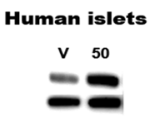
BIPIACTIN

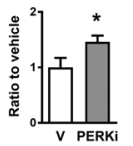

BiPIACTIN

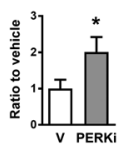

C

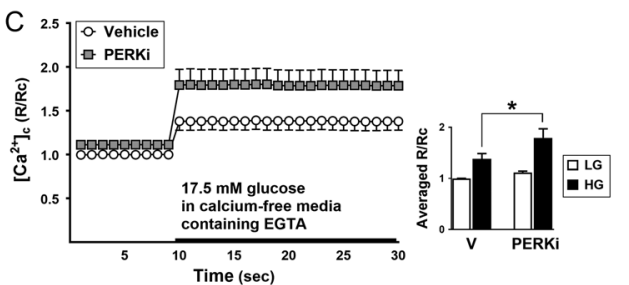

D

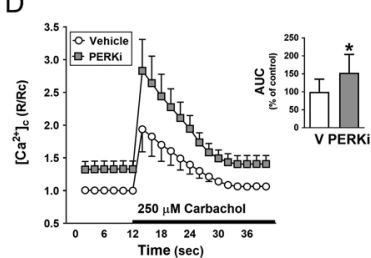

\section{Figure 2}

Low-dose GSK2606414 induced BiP expression, glucose-stimulated calcium release from intracellular stores and ER calcium. GSK2606414 was treated for $24 \mathrm{~h}$ to isolated islets at $40-50 \mathrm{nM}$. (A) Transcripts of UPR genes, relative to Gapdh, were assessed by quantitative RT-PCR $(n=5-6)$. (B) Representative pictures of western blotting for BiP using mouse (left, $n=7$ ) and human islets (right, $n=4$ ) and the quantitative analyses. (C) Glucose-stimulated calcium transit from intracellular calcium stores in calcium-free media containing EGTA. Cytosolic calcium concentrations (R) were expressed as relative ratios to the control before stimulation (Rc) $(n=6)$. For statistical analysis, the R/Rc measures were averaged as in the inset. (D) Cytosolic calcium released from ER by carbachol (R), expressed as relative ratios to the control before stimulation (Rc) $(n=6)$. For statistical analysis, AUC above each group's baseline were compared as in the inset. Paired $t$-test and 2-way repeated-measures ANOVA with Bonferroni posttests were applied. AUC, area under the curve; HG, high glucose; LG, low glucose; PERKi, PERK inhibitor; UPR, unfolded protein response; $\mathrm{V}$, vehicle. ${ }^{*} P<0.05$ vs vehicle.

\section{Low-dose GSK2606414 enhanced ER calcium} contents, islet insulin contents and GSIS in a Bipdependent manner

We used Bip siRNA (siBip) to investigate the role of BiP in GSK2606414-enhanced GSIS in dispersed mouse islet cells. When GSK2606414-induced Bip expression was suppressed (Fig. 3A), the GSK2606414-induced ER calcium level by carbachol stimulation decreased compared to that in the cells transfected with negative control siRNA (Fig. 3B).

We inhibited ER calcium refilling using thapsigargin, an inhibitor of sarcoendoplasmic reticulum calcium transport ATPase (SERCA) and measured the subsequent increase in cytosolic calcium to estimate calcium leakage form the ER (Schorr et al. 2015). Under those conditions, siBip abrogated the effects of GSK2606414 on ER calcium leakage (Fig. 3C), glucose-stimulated calcium transit (Fig. 3D), GSIS (Fig. 3E) and the insulin contents of the islet cells (Fig. 3F). When the GSIS was adjusted by the insulin contents, Bip expression no longer affected the GSK2606414-enhanced GSIS (Fig. 3G, left panel), but siBip reduced the ratio of insulin secretion between the high glucose condition and the low glucose condition (Fig. 3G, right panel). Those findings suggest that BiP plays a role in the effects of GSK2606414 on the insulin contents and exocytosis in islet cells and that the regulation of ER calcium contents by $\mathrm{BiP}$ might have an influence on the latter.

\section{Low-dose GSK2656157 improved GSIS in vivo}

We administered low-dose GSK2656157 (5 mg/kg twice per day) for 8 weeks to male wild-type and Atg7 $\Delta \beta$ cell ( $\beta$ cell-specific autophagy deficient) C57BL/6J mice. $\beta$ cell-specific autophagy deficiency has been implicated in $\beta$ cell function and viability (Jung et al. 2008) and especially in vulnerability to human islet amyloid polypeptide (IAPP)-induced toxicity (Kim et al. 2014, Rivera et al. 2014, Shigihara et al. 2014). The GSK2656157 treatment did not influence the fed blood glucose levels in the wild-type mice but reduced them in the $\operatorname{Atg} 7 \Delta \beta$ cell mice (Fig. 4A), without having any influence on body weight (Fig. 4B). GTTs revealed that the GSK2656157 treatment significantly improved the glucose tolerance in both mouse models (Fig. 4C). Measurements of GSIS in vivo suggested that those improvements could come from the augmentation of GSIS by GSK2656157 (Fig. 4D). The GSK2656157 treatment also increased the insulin sensitivity in the $\operatorname{Atg} 7 \Delta \beta$ cell mice (Fig. 4E).

\section{Discussion}

Unlike the complete blocking of PERK, the partial inhibition of PERK by the synthetic inhibitors GSK2606414 and GSK2656157 enhanced GSIS from pancreatic islets and reduced blood glucose levels in mice. The enhanced GSIS was associated with increases in cytosolic calcium 


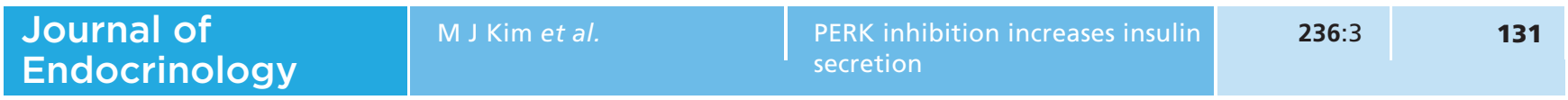

A

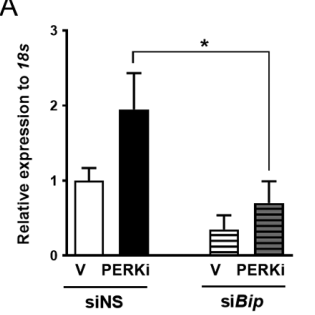

C

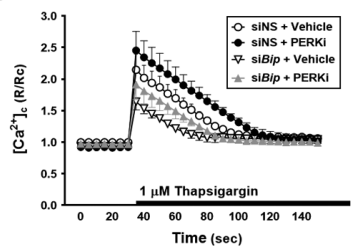

$E$

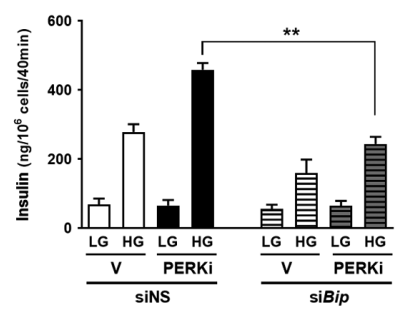

G
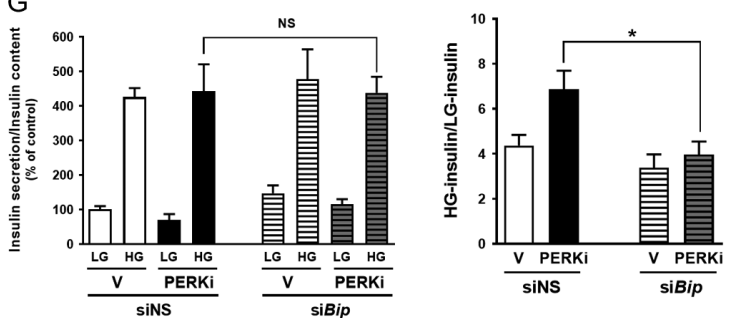

B

D
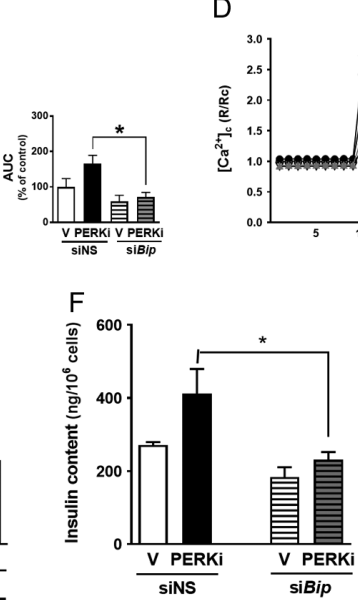
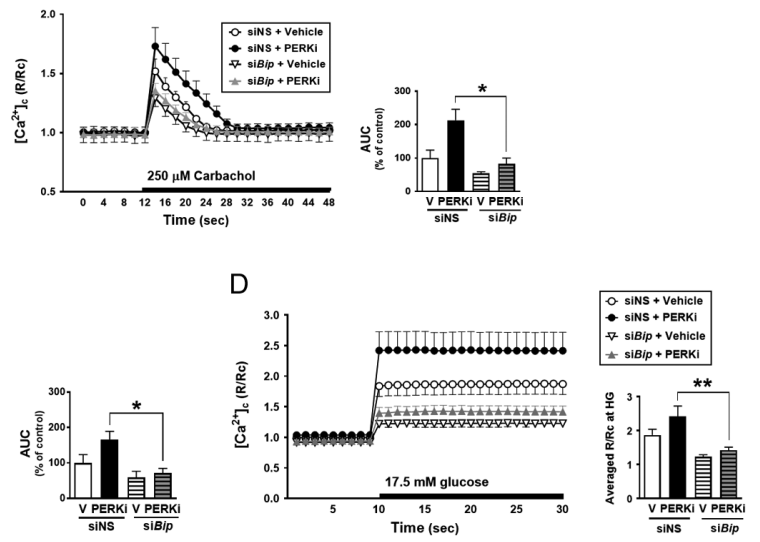

transit, ER calcium and islet insulin contents. Those effects of PERK inhibition were mediated by BiP induction.

GSK2606414, a highly potent and selective PERK inhibitor developed with a structure-based design, binds the kinase domain of PERK and competes with ATP (Axten et al. 2012). A previous study showed that the half maximal inhibitory concentration $\left(\mathrm{IC}_{50}\right)$ of GSK2606414 for PERK was 17-55 nM and that $1000 \mathrm{nM}$ GSK2606414 acutely blocked EIF2A phosphorylation in $\beta$ cells under ER stress, causing the inhibition of the UPR, the persistence of protein synthesis and the accumulation of misfolded proinsulin (Harding et al. 2012). We observed similar effects of GSK2606414 at and above $250 \mathrm{nM}$, along with significant reductions in EIF2A phosphorylation and GSIS (Fig. 1A and B). Our in vitro observations uncoupled the latent role of PERK in $\beta$ cell development from the roles of PERK in the regulation of insulin secretory function, just like Harding and coworkers had done in the regulation of unfolded protein flux (Harding et al. 2012).

Figure 3

Low-dose GSK2606414 effects were dependent on Bip expression. Mouse islets were dissociated into single cells, transfected with siBip or siNS and treated with $40 \mathrm{nM}$ GSK2606414 for $24 \mathrm{~h}$.

(A) mRNA levels of Bip relative to $18 \mathrm{~s}(n=6)$.

(B) Cytosolic calcium released from ER by carbachol $(R)$, expressed as relative ratios to the control before stimulation (Rc). AUC above each baseline was calculated for statistical analysis as the inset $(n=4)$. (C) Cytosolic calcium leaked from ER by thapsigargin $(R)$, expressed as relative ratios to the control before stimulation (Rc). AUC above each baseline was calculated for statistical analysis as the inset $(n=4)$. (D) Glucose-stimulated calcium transit. Cytosolic calcium concentrations (R) were expressed as relative ratios to the control before stimulation (Rc). For statistical analysis, the $\mathrm{R} / \mathrm{Rc}$ measures during high glucose were averaged as the inset $(n=9)$. (E) GSIS $(n=5)$, (F) insulin contents $(n=4)$ and (G) GSIS adjusted by insulin contents ( $n=4$, left panel), and the ratio of it to LG insulin levels (right panel). Two-way ANOVA with Bonferroni posttests was applied. AUC, area under the curve; $H G$, high glucose; $L G$, low glucose; NS, no significant difference; PERKi, PERK inhibitor; siBip, Bip siRNA; siNS, negative control siRNA; $V$, vehicle. ${ }^{*} P<0.05 ; * * P<0.01$ vs sins.
Low-dose GSK2606414 had effects on GSIS that were opposite to those of high-dose GSK2606414 (Fig. 1A). There is a recent report that a small-molecule ATP mimic with APY29 scaffold inhibited PERK at high concentration but, paradoxically, activated it at low concentrations, resulting in a bell-shaped activation profile (Mendez et al. 2015). The author presumed that partial occupancy of the ATP-binding site by the inhibitor biased the PERK kinase toward its active conformation. The paradoxical activation of kinase signaling by kinase inhibitors had been first noted in Raf kinase inhibitors (Hall-Jackson et al. 1999). Although GSK2606414 downregulated PERK phosphorylation, we did not measure the oligomerization of PERK monomers, which is associated with BiP release. So, there is a possibility that low-dose GSK2606414 did not attenuate but instead activated PERK (that is, oligomerization).

When we treated islets with $40 \mathrm{nM}$ GSK2606414 for 24h, PERK phosphorylation was sufficiently reduced, 

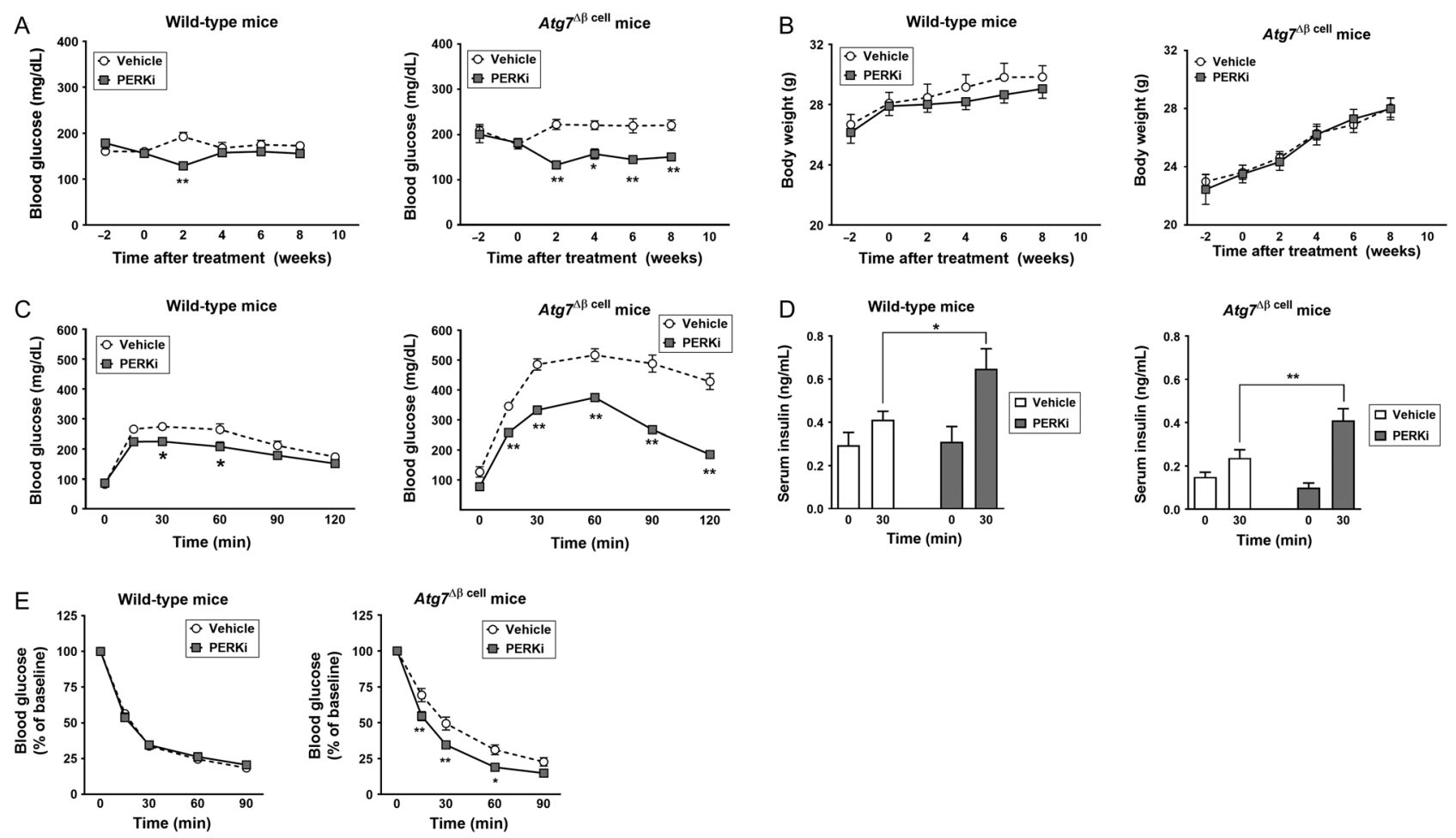

\section{Figure 4}

Low-dose GSK2656157 enhanced GSIS and improved hyperglycemia in vivo. GSK2656157 (5 mg/kg twice a day) and the vehicle were administered for

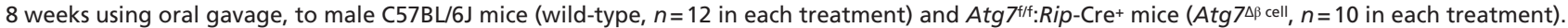
(A) Fed blood glucose levels, (B) body weights, (C) glucose tolerance test, (D) serum insulin levels before and 30 min after glucose loading and (E) insulin tolerance test. Two-way repeated-measures ANOVA with Bonferroni posttests were used. PERKi, PERK inhibitor; $\mathrm{V}$, vehicle. ${ }^{*} P<0.05 ; * \star P<0.01$ vs vehicle.

but there was no significant change either in EIF2A phosphorylation (Fig. 1B) or in downstream Atf4 transcription and the final UPR outcome Chop (Fig. 2A). Therefore, chronic and partial inhibition of PERK without additional ER stress by low-dose GSK2606414 in our study might work through another pathway from the well-known UPR pathway through EIF2A. It is possible that kinases other than PERK within the EIF2AK family (EIF2AK1, EIF2AK2 and EIF2AK4) compensated for the attenuation of PERK (EIF2AK3), especially in light of the fact that $1000 \mathrm{nM}$ GSK2606414 completely abrogated PERK phosphorylation while allowing partial phosphorylation of EIF2A. Although each EIF2AK family member is regulated specifically by its activator(s) and inhibitor(s), some mediators (e.g., NO (Tong et al. 2011)) seem to connect the different EIF2AK signaling pathways to one another.

The non-significant change of EIF2A phosphorylation by low-dose GSK2606414 (Fig. 1B) suggests that low-dose GSK2606414 did not affect the regulation of insulin translation by EIF2A. Rather, the effects of GSK2606414 on the insulin contents of the islet cells were dependent on Bip expression (Fig. 3F). BiP appears to have several functions in peptide transport into the ER after translation as well as in protein folding and assembly in the ER (Dudek et al. 2009). Therefore, it is possible that increased BiP expression caused by GSK2606414 increases the post-translational efficiency of insulin synthesis. To test that hypothesis, further proteostasis studies of BiP will be required.

In contrast to our results, Teodoro-Morrison and coworkers reported that the $\beta$ cell-specific overexpression of human BIP/GRP78 in mice did not change the islet insulin contents (Teodoro-Morrison et al. 2013). In addition, when transgenic mice were fed a high-fat diet (HFD), their islet insulin contents were lower than those in non-transgenic littermates that were fed the same HFD. Interestingly, those $\beta$ cell-specific transgenic mice were less obese, less insulin resistant and more glucose tolerant than their nontransgenic littermates. As a result, when fed an HFD, the transgenic mice were less hyperinsulinemic than the wildtype littermates. The authors suggested that BIP/GRP78 overexpression in $\beta$ cells protects the islets from ER stress induced by an HFD, maintaining a more normal structure and better function of islets. However, the mechanisms of those effects could not be explained. In addition, it is 
possible that the unexplained improvement in peripheral insulin resistance in the transgenic mice relieved the islets. Therefore, with the potential confounding effects on the islets of insulin resistance, it is difficult to conclude whether BiP overexpression enhances or suppresses islet insulin contents in vivo.

Bip was the only UPR gene that we observed to be affected by low-dose GSK2606414. As an ER chaperone, BiP directly interacts with PERK (Dudek et al. 2009): PERK monomers cannot be dimerized and stay inactive when $\mathrm{BiP}$ is bound to their luminal domain. When BiP becomes sequestered by unfolded polypeptides, it is released from PERK, allowing PERK to oligomerize and become active as a signal transducer (Fig. 5). Increased Bip expression has been observed not only in insulin-producing cells in which Perk was acutely ablated in vitro (Feng et al. 2009) but also in islets from Perk ${ }^{+/-}$mice (Wang et al. 2014). In the Perk ${ }^{+/}$mice, the Bip induction was transient during the early post-natal period (day 17) and accompanied by a transient increase in serum insulin (Wang et al. 2014). The mechanisms by which PERK insufficiency induces BiP expression are yet to be elucidated; however, PERK activation was reported to induce BiP expression through ATF6 (Luo et al. 2003).

Overexpression of BiP in INS1 cells appeared to have protective effects against human IAPP-induced ER stress (Cadavez et al. 2014). $\beta$ cell-specific overexpression of human BIP in mice also had protective effects against ER stress caused by a HFD (Teodoro-Morrison et al. 2013). In both studies, the protective effects of BiP were accompanied by improved GSIS. If BiP mediated the effects of GSK2606414 on islets (Fig. 3), then PERKi could

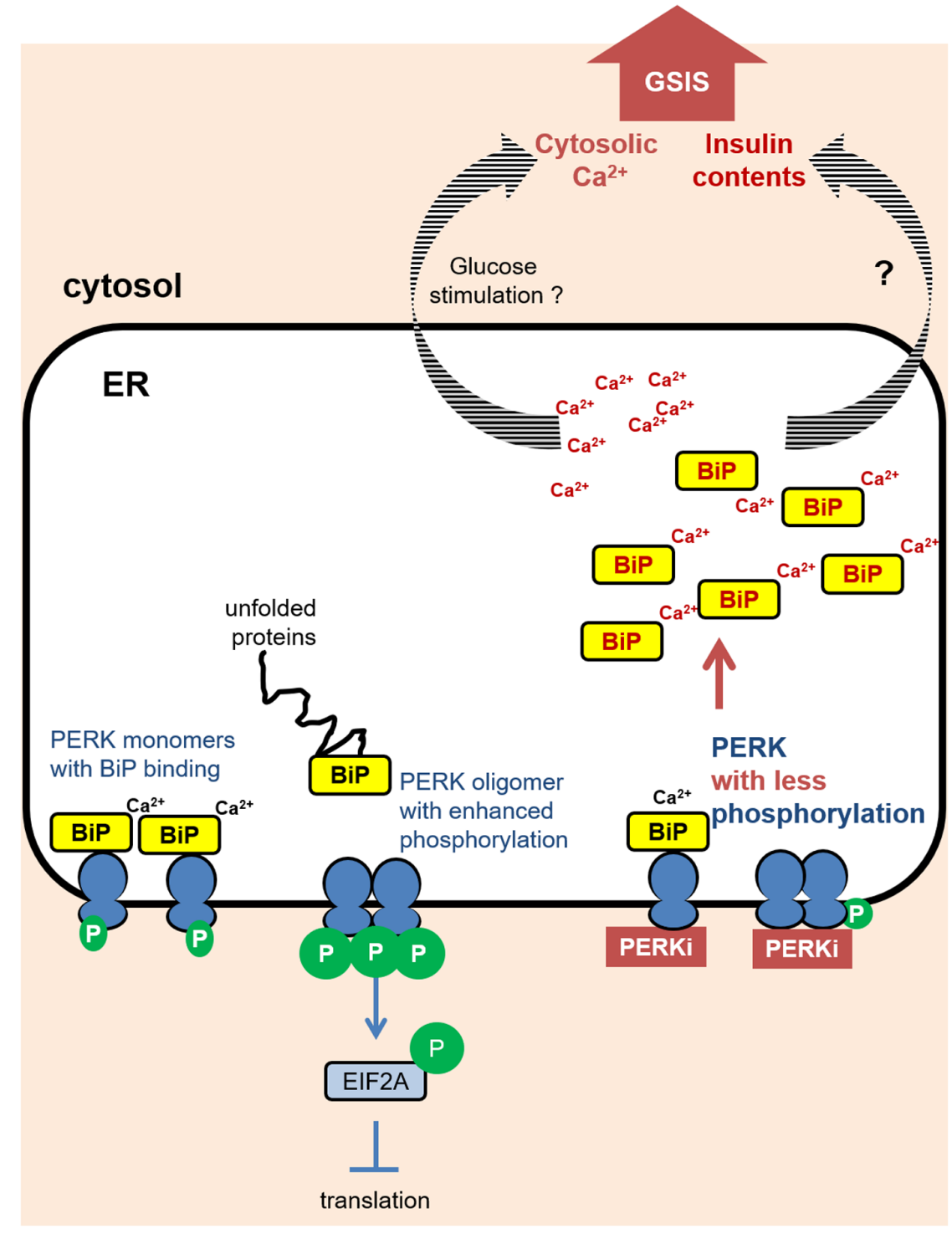

Figure 5

A working hypothesis of low-dose PERKi in pancreatic $\beta$ cells. GSK2606414 inhibits autophosphorylation of PERK by binding to its cytosolic domain, in competition with ATP. It induces BiP expression independently of EIF2A. Induced BiP increases ER calcium contents, and it may contribute to glucose-stimulated cytosolic calcium. Induced BiP also facilitates islet insulin contents. Both the BiP-mediated phenomena enhance GSIS. 
be used as an agent to enhance BiP, which seems to have favorable effects in $\beta$ cells under metabolic stress.

BiP also regulates cellular calcium homeostasis (Dudek et al. 2009). BiP is one of the calcium-binding proteins in the ER and has a direct role in the control of calcium ion efflux from the ER (Dudek et al. 2015). Additionally, Bip possesses calcium-responsive promoter elements, which are activated by ER calcium depletion (Li et al. 1993). Therefore, we hypothesized that the Bip transcription induced by low-dose GSK2606414 increased the ER calcium contents and contributed to glucose-stimulated cytosolic calcium transit and insulin secretion. Although we showed an association between the GSK2606414induced ER calcium contents and glucose-stimulated cytosolic calcium transit (Figs $1 \mathrm{E}$ and 2C, D) and both were Bip dependent (Fig. 3B and C), the evidence of a causal relationship between them is still weak. In addition, the mechanisms by which PERK and BiP interact to increase the ER calcium and also any influences of BiP on ER calcium regulators such as SERCA and ER calcium channels, should be investigated further. Wang and coworkers reported that acute ablation (20 min) of PERK with 1000 nM GSK2606414 did not affect the ER calcium contents but inhibited store-operated calcium entry and SERCA activity in concert with calcineurin, causing the acute ablation of calcium dynamics and GSIS (Wang et al. 2013). When we added GSK2606414 to the media used to measure cytosolic calcium and GSIS to test whether acute relief from low-dose GSK2606414 during GSIS (for $100 \mathrm{~min}$ ) after a 24-h exposure could affect our results, there was no difference in cytosolic calcium transit and GSIS (data not shown). Therefore, PERK inhibition seems to have different effects on calcium regulation depending on its degree and duration.

Finally, we showed that low-dose GSK2656157 could increase insulin secretion in vivo not only in wild-type mice with normoglycemia but also in $\beta$ cell-specific autophagydeficient hyperglycemic mice (Fig. 4D). Insulin sensitivity also improved in the latter (Fig. 4E), suggesting a direct effect of GSK2656157 on insulin target organs as well as an indirect effect in the form of improved hyperglycemia resulting from enhanced GSIS. Whether GSK2656157 is an insulin sensitizer or not is another topic for further research (Bobrovnikova-Marjon et al. 2012, Zhang et al. 2013, Gu et al. 2015). Considering the possibility for clinical application, additional experiments on $\beta$ cell mass and pancreatic volume should be performed, because high-dose GSK2656157 (more than 10 times higher than the effective dose used in our study) has been reported to reduce pancreatic volume in vivo (Atkins et al. 2013).
In addition, in the case of increased ER stress such as that in DM, even low-dose PERKi might profoundly affect the UPR, which can deteriorate cell viability. Favorable effects might be observed depending on the phenotype of Perk $^{+/-}$mice, which showed enhanced $\beta$ cell proliferation and substantially increased $\beta$ cell mass in the adult stage (Wang et al. 2014).

In summary, low-dose PERKi chronically inhibited the auto-phosphorylation of PERK and induced BiP expression (Fig. 5). The induction of BiP mediated the PERKi-induced changes in islet insulin contents and glucose-stimulated cytosolic calcium transit. The enhancement of ER calcium homeostasis is a putative mechanism for the cytosolic calcium transit. As a result, low-dose PERKi contributed to GSIS. Although we did not examine it, $\beta$ cell proliferation could be another contributor, based on previous results from Perk heterozygous mice (Wang et al. 2014). PERK is well known as an ER stress sensor that triggers the UPR, but it seems to have another role in insulin secretion through BiP in pancreatic islets. Our results demonstrate a relationship between PERK and BiP that influences the regulation of calcium homeostasis and insulin secretion, suggesting the possibility that low-dose PERKi could be used as a therapeutic approach to treat insulin deficiency.

\section{Supplementary data}

This is linked to the online version of the paper at https://doi.org/10.1530/ JOE-17-0497.

\section{Declaration of interest}

The authors declare that there is no conflict of interest that could be perceived as prejudicing the impartiality of the research reported.

\section{Funding}

This work was supported by the Innovative Research Institute for Cell Therapy of the Ministry of Health and Welfare, Republic of Korea, (grant number A062260) and the Seoul National University Hospital (gran number 03-2012-0380). The funders hand no role in study design, data collection and interpretation or the decision to submit the work for publication.

\section{Author contribution statement}

$\mathrm{M} \mathrm{J} \mathrm{K} \mathrm{and} \mathrm{H} \mathrm{S} \mathrm{J} \mathrm{designed} \mathrm{the} \mathrm{study,} \mathrm{researched} \mathrm{data} \mathrm{and} \mathrm{wrote} \mathrm{the}$ manuscript. S H M, J Y J and S W K provided human islets and collected clinical data. S Y S and M N K performed experiments. $\mathrm{H} \mathrm{L}$ and K S P discussed data and reviewed the manuscript. $\mathrm{H} \mathrm{S} \mathrm{J} \mathrm{is} \mathrm{the} \mathrm{guarantor} \mathrm{of} \mathrm{this}$ work and, as such, had full access to all the data in the study and takes responsibility for the integrity of the data and the accuracy of the data analysis. 


\section{Acknowledgments}

This study was supported by a grant from the Innovative Research Institute for Cell Therapy (A062260) by the Ministry of Health and Welfare, Republic of Korea, and the research grant from the Seoul National University Hospital (03-2012-0380). The authors are grateful to Prof. Masaaki Komatsu (Tokyo Metropolitan Institute of Medical Science, Japan) for the kind donation of Atg7-floxed mice.

\section{References}

Atkins C, Liu Q, Minthorn E, Zhang SY, Figueroa DJ, Moss K, Stanley TB, Sanders B, Goetz A, Gaul N, et al. 2013 Characterization of a novel PERK kinase inhibitor with antitumor and antiangiogenic activity. Cancer Research 73 1993-2002. (https://doi.org/10.1158/0008-5472. CAN-12-3109)

Axten JM, Medina JR, Feng Y, Shu A, Romeril SP, Grant SW, Li WH, Heerding DA, Minthorn E, Mencken T, et al. 2012 Discovery of 7-methyl-5-(1-\{[3-(trifluoromethyl)phenyl]acetyl\}-2,3-dihydro-1Hindol-5-yl)-7H-p yrrolo[2,3-d]pyrimidin-4-amine (GSK2606414), a potent and selective first-in-class inhibitor of protein kinase $\mathrm{R}$ (PKR)-like endoplasmic reticulum kinase (PERK). Journal of Medicinal Chemistry 55 7193-7207. (https://doi.org/10.1021/jm300713s)

Axten JM, Romeril SP, Shu A, Ralph J, Medina JR, Feng Y, Li WH, Grant SW, Heerding DA, Minthorn E, et al. 2013 Discovery of GSK2656157: an optimized PERK inhibitor selected for preclinical development. ACS Medicinal Chemistry Letters 4 964-968. (https://doi. $\operatorname{org} / 10.1021 / \mathrm{ml} 400228 \mathrm{e})$

Back SH \& Kaufman RJ 2012 Endoplasmic reticulum stress and type 2 diabetes. Annual Review of Biochemistry 81 767-793. (https://doi. org/10.1146/annurev-biochem-072909-095555)

Bobrovnikova-Marjon E, Pytel D, Riese MJ, Vaites LP, Singh N, Koretzky GA, Witze ES \& Diehl JA 2012 PERK utilizes intrinsic lipid kinase activity to generate phosphatidic acid, mediate Akt activation, and promote adipocyte differentiation. Molecular and Cellular Biology 32 2268-2278. (https://doi.org/10.1128/MCB.00063-12)

Cadavez L, Montane J, Alcarraz-Vizan G, Visa M, Vidal-Fabrega L, Servitja JM \& Novials A 2014 Chaperones ameliorate beta cell dysfunction associated with human islet amyloid polypeptide overexpression. PLOS ONE 9 e101797. (https://doi.org/10.1371/ journal.pone.0101797)

Delepine M, Nicolino M, Barrett T, Golamaully M, Lathrop GM \& Julier C 2000 EIF2AK3, encoding translation initiation factor 2-alpha kinase 3 , is mutated in patients with Wolcott-Rallison syndrome. Nature Genetics 25 406-409. (https://doi.org/10.1038/78085)

Dudek J, Benedix J, Cappel S, Greiner M, Jalal C, Muller L \& Zimmermann R 2009 Functions and pathologies of BiP and its interaction partners. Cellular and Molecular Life Sciences 66 1556-1569. (https://doi.org/10.1007/s00018-009-8745-y)

Dudek J, Pfeffer S, Lee PH, Jung M, Cavalie A, Helms V, Forster F \& Zimmermann R 2015 Protein transport into the human endoplasmic reticulum. Journal of Molecular Biology 427 1159-1175. (https://doi. org/10.1016/j.jmb.2014.06.011)

Feng D, Wei J, Gupta S, McGrath BC \& Cavener DR 2009 Acute ablation of PERK results in ER dysfunctions followed by reduced insulin secretion and cell proliferation. BMC Cell Biology 10 61. (https://doi. org/10.1186/1471-2121-10-61)

Gao Y, Sartori DJ, Li C, Yu QC, Kushner JA, Simon MC \& Diehl JA 2012 PERK is required in the adult pancreas and is essential for maintenance of glucose homeostasis. Molecular and Cellular Biology 32 5129-5139. (https://doi.org/10.1128/MCВ.01009-12)

Gu N, Guo Q, Mao K, Hu H, Jin S, Zhou Y, He H, Oh Y, Liu C \& Wu Q 2015 Palmitate increases musclin gene expression through activation of PERK signaling pathway in $\mathrm{C} 2 \mathrm{C} 12$ myotubes. Biochemical and
Biophysical Research Communications 467 521-526. (https://doi. org/10.1016/j.bbrc.2015.10.005)

Gupta S, McGrath B \& Cavener DR 2010 PERK (EIF2AK3) regulates proinsulin trafficking and quality control in the secretory pathway. Diabetes 59 1937-1947. (https://doi.org/10.2337/db09-1064)

Hall-Jackson CA, Eyers PA, Cohen P, Goedert M, Boyle FT, Hewitt N, Plant H \& Hedge P 1999 Paradoxical activation of Raf by a novel Raf inhibitor. Chemistry and Biology 6 559-568. (https://doi.org/10.1016/ S1074-5521(99)80088-X)

Harding HP, Zeng H, Zhang Y, Jungries R, Chung P, Plesken H, Sabatini DD \& Ron D 2001 Diabetes mellitus and exocrine pancreatic dysfunction in perk-/- mice reveals a role for translational control in secretory cell survival. Molecular Cell 7 1153-1163. (https://doi. org/10.1016/S1097-2765(01)00264-7)

Harding HP, Zyryanova AF \& Ron D 2012 Uncoupling proteostasis and development in vitro with a small-molecule inhibitor of the pancreatic endoplasmic reticulum kinase, PERK. Journal of Biological Chemistry 287 44338-44344. (https://doi.org/10.1074/jbc. M112.428987)

Henquin JC 2000 Triggering and amplifying pathways of regulation of insulin secretion by glucose. Diabetes 49 1751-1760. (https://doi. org/10.2337/diabetes.49.11.1751)

Jung HS, Chung KW, Won Kim J, Kim J, Komatsu M, Tanaka K, Nguyen YH, Kang TM, Yoon KH, Kim JW, et al. 2008 Loss of autophagy diminishes pancreatic beta cell mass and function with resultant hyperglycemia. Cell Metabolism 8 318-324. (https://doi. org/10.1016/j.cmet.2008.08.013)

Kaufman RJ 2002 Orchestrating the unfolded protein response in health and disease. Journal of Clinical Investigation 110 1389-1398. (https://doi.org/10.1172/JCI0216886)

Kim J, Cheon H, Jeong YT, Quan W, Kim KH, Cho JM, Lim YM, Oh SH, Jin SM, Kim JH, et al. 2014 Amyloidogenic peptide oligomer accumulation in autophagy-deficient beta cells induces diabetes. Journal of Clinical Investigation 124 3311-3324. (https://doi. org/10.1172/JCI69625)

Li WW, Alexandre S, Cao X \& Lee AS 1993 Transactivation of the grp78 promoter by $\mathrm{Ca} 2+$ depletion. A comparative analysis with $\mathrm{A} 23187$ and the endoplasmic reticulum $\mathrm{Ca}(2+)$-ATPase inhibitor thapsigargin. Journal of Biological Chemistry 268 12003-12009.

Luo S, Baumeister P, Yang S, Abcouwer SF \& Lee AS 2003 Induction of Grp78/BiP by translational block: activation of the Grp78 promoter by ATF4 through and upstream ATF/CRE site independent of the endoplasmic reticulum stress elements. Journal of Biological Chemistry 278 37375-37385. (https://doi.org/10.1074/jbc.M303619200)

Mendez AS, Alfaro J, Morales-Soto MA, Dar AC, McCullagh E, Gotthardt K, Li H, Acosta-Alvear D, Sidrauski C, Korennykh AV, et al. 2015 Endoplasmic reticulum stress-independent activation of unfolded protein response kinases by a small molecule ATP-mimic. eLife 4 e05434. (https://doi.org/10.7554/eLife.05434)

Rivera JF, Costes S, Gurlo T, Glabe CG \& Butler PC 2014 Autophagy defends pancreatic beta cells from human islet amyloid polypeptideinduced toxicity. Journal of Clinical Investigation 124 3489-3500. (https://doi.org/10.1172/JCI71981)

Schorr S, Klein MC, Gamayun I, Melnyk A, Jung M, Schauble N, Wang Q, Hemmis B, Bochen F, Greiner M, et al. 2015 Co-chaperone specificity in gating of the polypeptide conducting channel in the membrane of the human endoplasmic reticulum. Journal of Biological Chemistry 290 18621-18635. (https://doi.org/10.1074/jbc.M115.636639)

Shigihara N, Fukunaka A, Hara A, Komiya K, Honda A, Uchida T, Abe H, Toyofuku Y, Tamaki M, Ogihara T, et al. 2014 Human IAPP-induced pancreatic beta cell toxicity and its regulation by autophagy. Journal of Clinical Investigation 124 3634-3644. (https://doi.org/10.1172/ JCI69866)

Straub SG \& Sharp GW 2002 Glucose-stimulated signaling pathways in biphasic insulin secretion. Diabetes/Metabolism Research and Reviews 18 451-463. (https://doi.org/10.1002/dmrr.329)
() 2018 Society for Endocrinology Published by Bioscientifica Ltd. Printed in Great Britain 
Teodoro-Morrison T, Schuiki I, Zhang L, Belsham DD \& Volchuk A 2013 GRP78 overproduction in pancreatic beta cells protects against highfat-diet-induced diabetes in mice. Diabetologia 56 1057-1067. (https://doi.org/10.1007/s00125-013-2855-7)

Tong L, Heim RA \& Wu S 2011 Nitric oxide: a regulator of eukaryotic initiation factor 2 kinases. Free Radical Biology and Medicine $\mathbf{5 0}$ 1717-1725. (https://doi.org/10.1016/j.freeradbiomed.2011.03.032)

Wang R, McGrath BC, Kopp RF, Roe MW, Tang X, Chen G \& Cavener DR 2013 Insulin secretion and Ca2+ dynamics in beta-cells are regulated by PERK (EIF2AK3) in concert with calcineurin. Journal of Biological Chemistry 288 33824-33836. (https://doi.org/10.1074/jbc.M113.503664)

Wang R, Munoz EE, Zhu S, McGrath BC \& Cavener DR 2014 Perk gene dosage regulates glucose homeostasis by modulating pancreatic betacell functions. PLoS ONE 9 e99684. (https://doi.org/10.1371/journal. pone.0099684)
Zhang P, McGrath B, Li S, Frank A, Zambito F, Reinert J, Gannon M, Ma K, McNaughton K \& Cavener DR 2002 The PERK eukaryotic initiation factor 2 alpha kinase is required for the development of the skeletal system, postnatal growth, and the function and viability of the pancreas. Molecular and Cellular Biology 22 3864-3874. (https://doi.org/10.1128/MCB.22.11.3864-3874.2002)

Zhang W, Feng D, Li Y, Iida K, McGrath B \& Cavener DR 2006 PERK EIF2AK3 control of pancreatic beta cell differentiation and proliferation is required for postnatal glucose homeostasis. Cell Metabolism 4 491-497. (https://doi.org/10.1016/j. cmet.2006.11.002)

Zhang W, Hietakangas V, Wee S, Lim SC, Gunaratne J \& Cohen SM 2013 ER stress potentiates insulin resistance through PERK-mediated FOXO phosphorylation. Genes and Development 27 441-449. (https://doi. org/10.1101/gad.201731.112)

Received in final form 30 November 2017

Accepted 22 December 2017

Accepted Preprint published online 22 December 2017 http://joe.endocrinology-journals.org https://doi.org/10.1530/JOE-17-0497 (c) 2018 Society for Endocrinology Published by Bioscientifica Ltd. 